\title{
HARMONIC INTERACTION OF A STATIC VAR COMPENSATOR WITH AC POWER SYSTEM CONTAINING MULTIPLE NON-LINEAR LOADS
}

\author{
Mohamed Nassim Kraimia* - Mohamed Boudour
}

Department of Electrical Engineering, Laboratory of Electrical and Industrial Systems, University of Science and Technology Houari Boumediene, El Alia 16111, Bab Ezzouar, Algiers, Algeria.

\begin{tabular}{l} 
ARTICLE INFO \\
\hline Article history: \\
Received: 1.5 .2018$. \\
Received in revised form: 5.11 .2018$. \\
Accepted: 5.11 .2018$. \\
\hline Keywords: \\
Harmonic power flow \\
Static Var compensator \\
Harmonic interaction \\
Coupled current source \\
\hline DOI: http://doi.org/10.30765/er.40.3.03
\end{tabular}

\section{Introduction}

The progress in power electronic devices made possible the use of efficient reactive power compensating devices. These compensating devices belong to the Flexible Alternating Current transmission systems (FACTS) that include shunt and series compensating devices. The first one is used to improve the weak nodal voltage in power system either as a static Var compensator (SVC) or a static synchronous compensator (STATCOM) [1].

The static Var compensator has been used in transmission and distribution power systems as one of the pertinent solutions for power factor correction, reactive power compensation and weak nodal voltage improvement [2]. But these operations are done on the

\begin{abstract}
:
In this paper a study of the impact of the harmonics generated by a static Var compensator (SVC) is presented. The SVC is modeled, in the harmonic domain, as a coupled current source by using the complex Fourier transforms. Then, this model is converted to polar form to be integrated into the harmonic power flow program. This approach has been carried out on the IEEE 14 bus test power system, in order to show its effectiveness in evaluating the impact of harmonics, injected by the shunt compensating devices, and its interaction with the AC transmission system, in meshed power networks. Since the SVC consists of a thyristorcontrolled reactor (TCR) and a fixed capacitor, the harmonic currents are functions of the TCR thyristors firing angles. The variation of the total voltage harmonic distortion as function of firing angle changes and location of nonlinear loads is clearly presented and discussed.
\end{abstract}

determent of power quality, since the SVC contains in its circuit a thyristor-controlled reactor (TCR), in parallel with a fixed capacitor. Since the thyristorcontrolled reactor derives non-sinusoidal discontinuous current and consequently generates harmonic currents. It is considered as a high current source.

There has been much research conducted in the field of harmonics generation by the SVC. In [3] harmonic analysis of the SVC and STATCOM was performed using PSCAD/EMTDS simulations. In [4] a different scenario was investigated to determine harmonic resonance frequencies by using a harmonic impedance model of the power system in the presence of the SVC. A system changes impact of the existing SVC and its interaction with harmonics generated by wind generation installed at the same bus [5]. A

\footnotetext{
* Corresponding author. Tel.: +213- 662-15-49-34.

E-mail address: kraimia.nassim@yahoo.fr
} 
comprehensive analysis on harmonic characteristics of different FC-TCR based SVC is reported in [6]. The authors presented in [7] a probabilistic approach for the SVC placement to control harmonic voltage distortion, as well as reactive power compensation in the power system.

The harmonic currents generated by nonlinear loads, flow into the power system and cause a distortion in the voltage and current waveforms. These harmonic currents could cause a resonance problem in electrical power systems. Thus, the operator may have information of nodal voltage and branch currents at each frequency. The harmonic power flow is a powerful tool to get the nodal harmonic voltages. In the last years, many researchers have been performed in the field of harmonic power flow algorithms. These algorithms vary in terms of data requirement and modeling complexity. In [8], the frequency response of network as seen from specified bus is determined using frequency scan algorithm. In [9-10], the conventional power flow is reformulated to include the nonlinear loads. This method was used to study such topics [1113] related to harmonics. In [14-15], the previous method is extended to include unbalance of the system. In [16], a model of the converter is established and included in the algorithm. A Norton equivalent model of nonlinear load is presented in [17].

With increasing of loads based on power electronics in the transmission power system, the total harmonic distortion voltage at bus system voltages will be very high. It is important to notice that the fixed current source model such that was used in [18] does not take into consideration the voltage harmonics changes. This is due to the fact that the harmonic current SVC is expressed as a function of the applied voltage and firing angle of TCR. The SVC can be modeled as coupled harmonic current source. In this paper, this model will be incorporated in the well-known harmonic power flow iterative algorithm [9] to show the harmonic interaction impact between the SVC harmonic current and AC electrical power system in presence of multiple nonlinear loads. This method has been carried out on the IEEE 14 bus test system, in the presence of five nonlinear loads producing odd harmonic components 5th; 7th; 11th; 13th; 17th; 19th; 23th; 25th in order to study harmonics related problem.

\section{The power system components modeling}

The first step in the harmonic power flow consists of modeling the linear components, such as transmission lines, generators and transformers, and the nonlinear loads at fundamental and harmonic frequencies.

Transmission line is modeled as lumped $\pi$ equivalent circuit as shown in fig.1. At the fundamental frequency, the admittance matrix is still the same as the one used in conventional power flow.

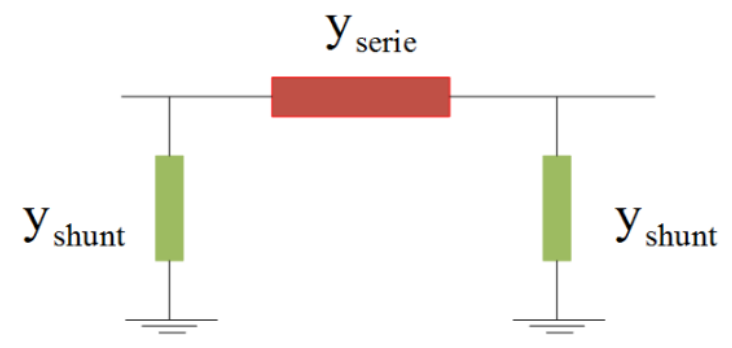

Figure 1. Transmission line model

However, at harmonic frequency, this admittance matrix is evaluated at each harmonic of interest and is given by:

$$
\bar{Y}^{(h)}=\left[\begin{array}{ccccc}
y_{11}^{(h)} & y_{12}^{(h)} & . & . & y_{1 n}^{(h)} \\
y_{21}^{(h)} & y_{22}^{(h)} & . & . & y_{2 n}^{(h)} \\
y_{31}^{(h)} & y_{32}^{(h)} & . & . & y_{31}^{(h)} \\
\vdots & \vdots & \vdots & \vdots & \vdots \\
y_{n 1}^{(h)} & y_{n 2}^{(h)} & . & . & y_{n n}^{(h)}
\end{array}\right]
$$

Generators are modeled as conventional PV buses with their lower and upper limits at fundamental frequency, while at harmonic frequency, it will be a passive element and its harmonic impedance is expressed as function of its sub-transient reactance as follows:

$$
\mathrm{X}_{g}^{(h)}=h \mathrm{X}_{g}^{\prime \prime}
$$

Where $X_{g}^{h}$ is the equivalent impedance at the $h^{\text {th }}$ harmonic order, and $X_{g}^{\prime \prime}$ is the sub-transient reactance of the generator. The transformer due to a nonlinear relation between the voltage and the current, its model will be very difficult at harmonic frequency. Moreover, it could be even considered as a harmonic producing device especially when operating under over excitation. In this paper we assume that the transformer is approximated by its linear leakage inductance under harmonic frequency and its appropriate harmonic impedance is: 


$$
\mathrm{Z}_{T}^{(h)}=R_{T}+J h X_{T}
$$

Capacitor and inductor are modeled as fixed shunt reactance and their harmonic impedance for the $h^{\text {th }}$ harmonic order are given by:

$$
\begin{gathered}
\mathrm{X}_{L}^{(h)}=2 \pi h f_{1} L \\
\mathrm{X}_{C}^{(h)}=1 /\left(2 \pi h f_{1} C\right)
\end{gathered}
$$

Where: $\mathrm{L}$ and $\mathrm{C}$ are the inductance and capacitance of inductor and capacitor respectively. $f_{1}$ is the fundamental frequency.

PQ and linear loads are modeled, at fundamental frequency, as conventional PQ or PV buses. Whereas at harmonic frequency, they are modeled as a shunt admittance given by the following expression:

$$
y_{k}^{(h)}=\frac{\left(P_{K}^{(1)}-j Q_{K}^{(1)}\right) / h}{\left|V_{K}^{(1)}\right|^{2}}
$$

\section{The basic circuit of the Static Var compensator}

The one-line diagram of the static Var compensator connected at bus $k$ is shown in Fig. 2. It consists of two parallel branches, including the thyristorcontrolled reactor and fixed capacitor. From the operating point of view, the SVC can be seen as a variable shunt reactance that derives a discontinuous inductive or capacitive current from the power system, depending on the operating conditions of the system. This current, rich in harmonic components, is generated by the TCR branch. The thyristorcontrolled reactor consists of two anti-parallel thyristors connected with a series reactor. These thyristors, Th1 and Th2, are fired sequentially in each positive and negative half cycle respectively. The firing angle $\alpha$ is measured from the positive zero crossing voltage, and since the fundamental current lags the source voltage in TCR branch by $90^{\circ}$ i.e. the TCR current control starts at $90^{\circ}$ when the supply voltage equals its maximum instantaneous value [19].

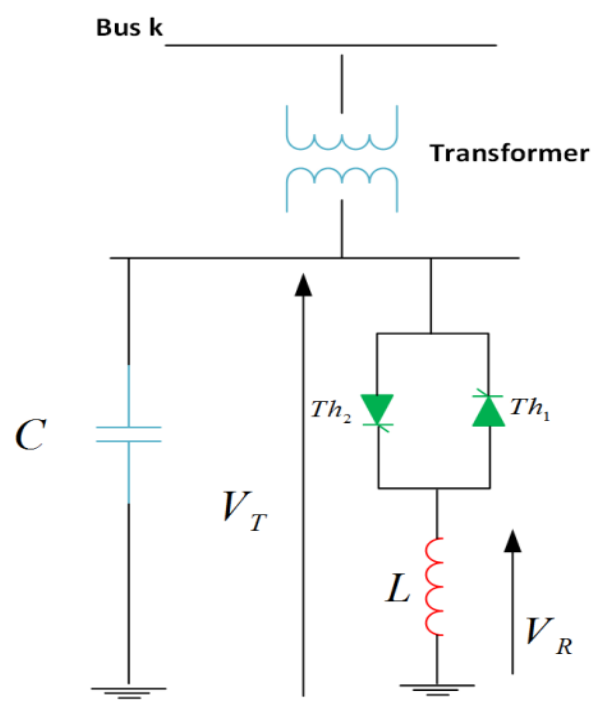

Figure 2. Basic circuit of the SVC

\section{The SVC model in the harmonic domain}

The switching function illustrating the sequential operation of the thyristors Th1 and Th2 is schematically represented in Fig.3. The state 1 indicates the turn on of one of the thyristors, while the state 0 indicates that both thyristors are turned off.

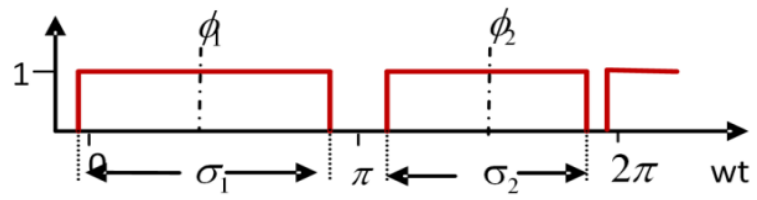

Figure 3. Switching function $H(t)$

If the bus voltage of the SVC is a periodic function and contains harmonic components, by using complex Fourier series we get its expression in the harmonic frequency domain as follows:

$$
\mathrm{V}_{\mathrm{T}}=\sum_{m=-\infty}^{+\infty} \mathrm{V}_{\mathrm{Tm}} e^{j(m w t)}
$$

Where: $\mathrm{V}_{\mathrm{Tm}}$ is the $\mathrm{m}^{\text {th }}$ harmonic complex coefficient of the applied bus voltage $\mathbf{V}_{\mathbf{k}}$. On the other hand, the switching function $\mathrm{H}(\mathrm{t})$, can be represented, in the harmonic domain, by the following expression:

$$
\mathrm{H}=\sum_{n=-\infty}^{+\infty} \mathrm{h}_{\mathrm{n}}\left(\sigma_{1}, \phi_{1}, \sigma_{2}, \phi_{2}\right) e^{j(n w t)}
$$


Where $h_{n}$ is $n^{\text {th }}$ harmonic coefficient of the switching function $\mathbf{H}$. From Fig. 3, $\sigma$ is the conduction angle and $\Phi$ is the centre of the conduction period. The harmonic magnitude of switching function is given by:

$$
\mathrm{h}_{\mathrm{n}}=\frac{1}{2 \pi} \int_{t 1}^{t 1+2 \pi} H(t) e^{-j(n w t)} d w t
$$

In the harmonic domain, the voltage across the inductor $\mathrm{V}_{\mathrm{R}}$ is given by:

$$
\mathrm{V}_{R}(t)=\mathrm{V}_{T} H=\sum_{m, n=-\infty}^{\infty} \mathrm{V}_{T m} h_{n} e^{j(m+n) w t}
$$

The product of the two complex phasors in (9) can be expressed as a matrix form, consisting of an Infinite magnitude of switching function multiplied by a phasor voltage vector of TCR.

$$
\left[\begin{array}{c}
\vdots \\
V_{R-3} \\
V_{R-2} \\
V_{R-1} \\
\vdots
\end{array}\right]=\left[\begin{array}{ccccc}
\cdot & \vdots & \vdots & \vdots & \cdot \\
\cdots & h_{0} & h_{-1} & h_{-2} & \cdots \\
\cdots & h_{1} & h_{0} & h_{-1} & \cdots \\
\cdots & h_{2} & h_{1} & h_{0} & \cdots \\
\cdot & \vdots & \vdots & \vdots & \cdot
\end{array}\right]\left[\begin{array}{c}
\vdots \\
V_{T-3} \\
V_{T-2} \\
V_{T-1} \\
\vdots
\end{array}\right]
$$

The $\mathbf{H}$ is a Toeplitz matrix that represents a crosscoupling between harmonics, which is an important characteristic in switching function. By referring to the models used in [20], the harmonic admittance matrices for the TCR and fixed capacitor are given as follow:

$$
\begin{aligned}
\mathrm{Y}_{\mathrm{TCR}} & =[L \mathrm{D}(J h w)]^{-1} \mathrm{H} \\
\mathrm{Y}_{\mathrm{C}} & =C[\mathrm{D}(J h w)]
\end{aligned}
$$

Where $\mathbf{D}$ is the differentiation matrix, $\mathrm{L}$ is the inductance of the series reactor of TCR and C is the capacitance of shunt capacitor. Thus, the equivalent admittance of the SVC in steady state can be written as:

$$
Y_{S V C}=Y_{T C R}+Y_{C}
$$

\subsection{Switching function calculation}

The calculation of the complex harmonic coefficients of the switching function in (8) needs a Newton iterative procedure since it is related to the applied voltage and the value of the delay firing angle $(\alpha)$. It should be noted that this formulation is more important since it could be applied even when the conduction period of two thyristors are not equal due to the asymmetry of half wave voltage waveform.

\subsection{Harmonic current characteristics of the SVC}

The current derived by the SVC, can be determined by a direct product of its harmonic admittance obtained in (13) and harmonic phasor voltage. This current is expressed in complex form, which contains positive and negative terms, including the harmonic interaction between SVC compensator and AC power system. The harmonic current injected into the system, has the same harmonic characteristics generated by the TCR i.e. are all odd harmonics but are different in phase and magnitude due to the shunt capacitor effect. Moreover, in the case where the secondary winding of the transformer is coupled in delta or ungrounded wye, triplens harmonics are not allowed to flow into the power system.

\section{Harmonic Power Flow Program}

There are a large number of harmonic analysis methods that are in widespread use. The most popular of these are: frequency scans, harmonic penetration and harmonic power flow and Current source (or current injection) methods [8], but these methods do not take into consideration the harmonic interaction with the power system components and harmonic sources. In this section, a harmonic power flow is illustrated based on the Newton Raphson method, since it has already been applied with harmonic source that has a V-I nonlinear characteristics. In this method we define linear bus as PQ or PV buses, and nonlinear bus is the bus at which the harmonic source is connected. However, the Newton's method is used for forcing the appropriate mismatch vector $(\Delta \mathbf{M})$ containing mismatch power $(\Delta \mathbf{W})$ and current $(\Delta \mathbf{I})$ to zero, using a Jacobean matrix $\mathbf{J}$ and obtaining appropriate correction terms $(\Delta \mathbf{U})$.

$$
\begin{array}{r}
\Delta M=[J] \Delta U^{\xi} \\
\Delta U^{\xi}=U^{\xi}-U^{\xi+1}
\end{array}
$$

Where $\zeta$ is the iteration number

The matrix formulation of the problem is: 


$$
\left[\begin{array}{l}
\overline{\Delta V}^{(1)} \\
\overline{\Delta V}^{(5)} \\
\overline{\Delta V}^{(7)} \\
\cdot \\
\cdot \\
\cdot \overline{\Delta V}^{(L)} \\
\overline{\Delta \varphi}
\end{array}\right]=\left[\begin{array}{ccccc}
\bar{J}^{(1)} & \bar{J}^{(5)} & \ldots & \bar{J}^{(L)} & 0 \\
\overline{Y G}^{(5,1)} & \overline{Y G}^{(5,5)} & \ldots & \cdot & \bar{H}^{(5)} \\
\overline{Y G}^{(7,1)} & \overline{Y G}^{(7,5)} & \ldots & \cdot & \bar{H}^{(7)} \\
\cdot & \cdot & \cdot & \cdot & \cdot \\
\cdot & \cdot & \cdot & \cdot & \cdot \\
\cdot & \cdot & \cdot & \cdot & \cdot \\
\overline{Y G}^{(L, 1)} & \overline{Y G}^{(L, 5)} & \ldots & \overline{Y G}^{(L, L)} & \bar{H}^{(L)} \\
\overline{Y G}^{(1,1)} & \overline{Y G}^{(1,5)} & \ldots & \overline{Y G}^{(1, L)} & \bar{H}^{(1)}
\end{array}\right]^{-1}\left[\begin{array}{l}
\overline{\Delta W} \\
\overline{\Delta I}^{(5)} \\
\overline{\Delta I}^{(7)} \\
\cdot \\
\cdot \\
\overline{\Delta I}^{(L)} \\
\overline{\Delta I}^{(1)}
\end{array}\right]
$$

\subsection{Mismatch power $(\Delta W)$}

The load flow equations used in the harmonic power flow are the same as the ones used in the fundamental power flow for all buses expect the nonlinear bus (at which the harmonic sources are connected) The difference being is in the nonlinear bus where additional equations are introduced for each harmonic frequency considered in the HPF in order to calculate the total harmonic real and reactive line current at nonlinear bus.

The expression of the fundamental and total power mismatch is given by:

$$
\overline{\Delta W}=\left[\begin{array}{l}
P_{2}^{(1)}+F_{r, 2}^{(1)}, Q_{2}^{(1)}+Q_{i, 2}^{(1)}, \ldots, P_{m-1}^{(1)}+F_{r, m-1}^{(1)}, Q_{r, m-1}^{(1)}+Q_{i, m-1}^{(1)}, \ldots, \\
\Delta P_{m}^{\text {nonlinear }}, \Delta Q_{m}^{\text {nonlinear }}, \Delta P_{n}^{\text {nonlinear }}, \Delta Q_{n}^{\text {nonlinear }}
\end{array}\right]
$$

Where, $\mathrm{P}_{\mathrm{j}}^{(1)}, \mathrm{Q}_{\mathrm{j}}{ }^{(1)}$ are the real and reactive fundamental load power for the linear bus $j$ and $F_{r, j}{ }^{(1)}$, $\mathrm{F}_{\mathrm{i}, \mathrm{j}}{ }^{(1)}$ are the line fundamental real and reactive powers for the linear bus j. $\Delta \mathrm{P}_{\mathrm{m}}{ }^{\text {nonlinear }}, \Delta \mathrm{Q}_{\mathrm{m}}{ }^{\text {nonlinear }}$ are the total real and reactive mismatches power for the nonlinear bus $\mathrm{m}$.

\subsection{Fundamental current Mismatch $(\Delta \mathrm{I}(1))$}

The expression of fundamental current mismatch is found by the application of the Kirchhoff current law at fundamental frequency for only nonlinear buses where all currents (e.g., fundamental real and reactive line currents $I_{r, m}{ }^{(l)}, I_{i, m}{ }^{(1)}$ and fundamental nonlinear real and reactive load currents $\left.G_{r, m}{ }^{(l)}, G_{i, m}{ }^{(1)}\right)$ are referred to the swing bus, the formulation is given by:

$$
\overline{\Delta I}^{(1)}=\left[I_{r, m}^{(1)}+G_{r, m}^{(1)}, I_{i, m}^{(1)}+G_{i, m}^{(1)}, \ldots, I_{r, n}^{(1)}+G_{r, n}^{(1)}, I_{i, n}^{(1)}+G_{i, n}^{(1)}\right]^{t}
$$

\subsection{Harmonic current Mismatch $(\Delta \mathrm{I}(1))$}

The harmonic current mismatch $\Delta \mathbf{I}^{(\mathbf{h})}$ is found by applying the Kirchhoff current law at harmonic frequency for all busses i.e is defined for linear and nonlinear buses including swing bus by:

$$
\begin{gathered}
\overline{\Delta I}^{(h)}= \\
=\left[\begin{array}{l}
I_{r, 1}^{(h)}, I_{i, 1}^{(h)}, \ldots, I_{r, m-1}^{(h)}, I_{i, m-1}^{(h)}, I_{r, m}^{(h)}+G_{r, m}^{(h)}, I_{i, m}^{(h)} \\
+G_{i, m}^{(h)}, \ldots, I_{r, n}^{(h)}+G_{r, n}^{(h)}, I_{i, n}^{(h)}+G_{i, n}^{(h)}
\end{array}\right]^{t}
\end{gathered}
$$

Where: $\mathrm{I}_{\mathrm{r}, \mathrm{m}}{ }^{(\mathrm{h})}, \mathrm{I}_{\mathrm{i}, \mathrm{m}}{ }^{\text {(h) }}$ are harmonic real and reactive line currents, $G_{r, m}{ }^{(h)}, G_{i, m}{ }^{(h)}$ harmonic nonlinear real and reactive load currents. The formulation of Jacobin matrix $\mathbf{J}$ is well described in [21].

\subsection{Integration of generating harmonic current in HPF}

The expression of the TCR current, as discussed in section 4 , is a complex form constraining negative and positive complex coefficients. In order to integrate it into HPF as coupled current source, it will 
be converted into its polar form by using the complex Fourier transform and then the current will be expressed by its magnitude and phase.

\section{The flow chart for Harmonic power flow including the SVC}

Solution procedure of the HPF with presence of the SVC can be presented as can be seen on Fig. 4.

\section{Case study and simulation results}

The solution procedure describing the integration of the SVC in the harmonic power flow is tested on the IEEE 14 bus transmission system [22] using Matlab package.

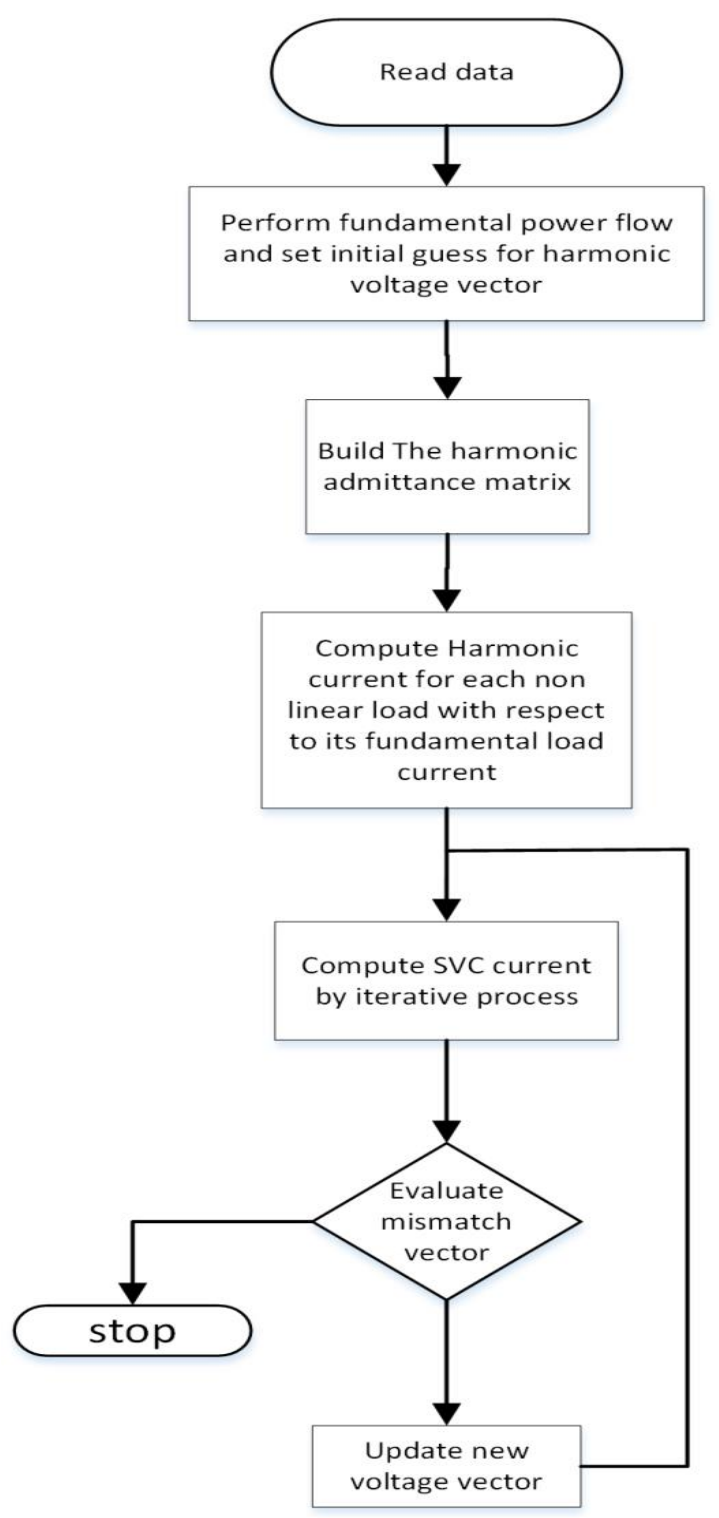

Figure 4. Flowchart for the method
The main step of this procedure consists of power system components modeling at fundamental and harmonic frequencies. The linear components of the tested system were modeled as PQ or PV bus at the fundamental frequency, while at the harmonic frequency they were modeled as passive elements whereas, the harmonic interaction impact of the SVC was investigated through two scenarios as following:

Scenario 01: The harmonic current interaction with AC system

In this scenario, the nonlinear load is connected at bus 12 as constant current source, and has a typical spectrum as tabulated in table A.1. The magnitude and phase angle corresponding to each harmonic frequency are determined with respect to the fundamental load current.

Since the delay firing angle varies from $90^{\circ}$ to $180^{\circ}$, the current generated by the SVC is also changing. Fig. 5. shows the variation of each harmonic current component of the SVC as a function of firing angle. We have noticed that the lower order harmonics, i.e. $5^{\text {th }} ; 7^{\text {th }} ; 11^{\text {th }}$ are predominant components but their peak instantaneous values differ.

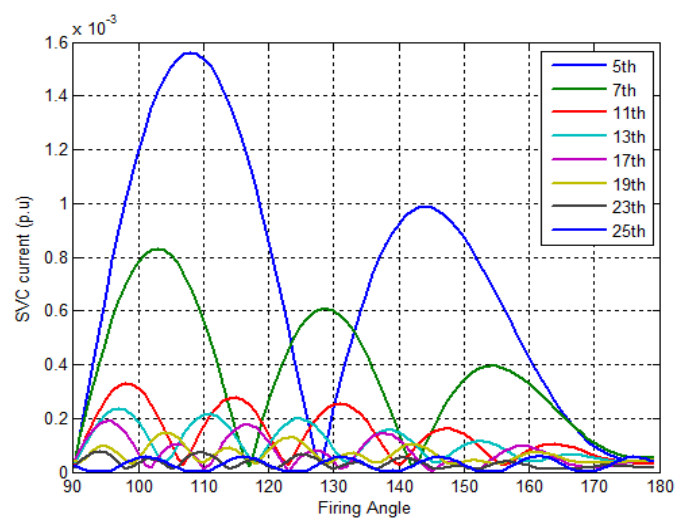

Figure 5. The harmonic current of the SVC connected at bus $N^{\circ} 12$

To illustrate the impact of the firing angle changing on the steady state waveform current, Fig.6-8 represents a set of current waveforms depicted in time domain for some fixed firing angles $90^{\circ}-120^{\circ}$ $160^{\circ}$ respectively. We notice that, the waveform shape changes as a function of the firing angle. Besides, both the thyristors are fully conducting at $90^{\circ}$. The current waveform shown in Fig. 6 has slowly distorted due to the harmonic interaction with the AC power system.

If the thyristors are controlled at $\alpha=120^{\circ}$, the current has a discontinuous form similar to one of the TCR, but there is some difference as depicted in Fig .7. due 
to the capacitor charge and discharge operations. It can be seen that its instantaneous peak is reduced from 0.03 (at $\left.\alpha=90^{\circ}\right)$ to $0.015\left(\alpha=120^{\circ}\right)$ and the $5^{\text {th }}$, $7^{\text {th }}$ and $11^{\text {th }}$ harmonics are still predominating components. From Fig. 8. when $\alpha=160^{\circ}$, the current waveform will be continuous, and it has a distorted waveform. All harmonic components have a negligible effect if the SVC operates at a firing angle close to $180^{\circ}$.

The results shown above have been found based on two iterative procedures. The first one is the Newton iterative method for obtaining the corresponding values of conduction period of each thyristor to determine the complex coefficients of switching function. The second one is the harmonic power flow method. However, two embedded iterative loops have been used in this paper to study the interaction impact of the SVC.

Scenario 02: Impact of the SVC with multiple nonlinear loads

In this case the tested system was modified by introduction of multiple nonlinear loads through five cases. In the beginning, the system was experienced with only the presence of the SVC connected at bus 14 , and then five nonlinear loads were connected at buses: 4, 5, 9, 11, and 13. The firing angle is set at $120^{\circ}$ for different cases. The number of connected non linear loads for each case is shown in table 01. In this table NLD signifies nonlinear load with spectrums data for each one tabulated in table A.2. The harmonic current derived by the SVC is affected by different cases in the sense that the SVC was

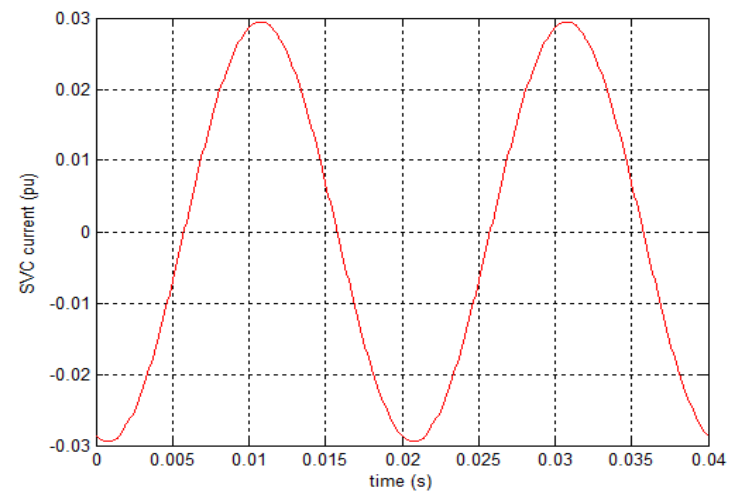

(a) modeled as coupled harmonic current source. This current is directly dependent on the applied voltage. The results are shown in figures 9 to 11 . Figure 9 illustrates total harmonic distortion of the voltage at the SVC bus. Figure 10 illustrates total harmonic distortion of the voltage at all busses. And finally, figure 11 illustrates the harmonic components of the SVC current.

Table 1. Five cases under study

\begin{tabular}{|c|c|c|c|c|c|}
\hline & $\begin{array}{c}\text { NLD } \\
\text { at bus } \\
04\end{array}$ & $\begin{array}{c}\text { NLD } \\
\text { at bus } \\
\text { 05 }\end{array}$ & $\begin{array}{c}\text { NLD } \\
\text { at bus } \\
\text { 09 }\end{array}$ & $\begin{array}{c}\text { NLD } \\
\text { at bus } \\
\mathbf{1 1}\end{array}$ & $\begin{array}{c}\text { NLD } \\
\text { at bus } \\
\mathbf{1 3}\end{array}$ \\
\hline Case 01 & \multicolumn{5}{|c|}{ Any Nonlinear loads } \\
\hline Case 02 & $\mathrm{X}$ & & & & \\
\hline Case 03 & $\mathrm{X}$ & $\mathrm{x}$ & & & \\
\hline Case 04 & $\mathrm{X}$ & $\mathrm{x}$ & $\mathrm{X}$ & & \\
\hline Case 05 & $\mathrm{X}$ & $\mathrm{x}$ & $\mathrm{x}$ & $\mathrm{x}$ & $\mathrm{X}$ \\
\hline
\end{tabular}

If the system operates only with the presence of the SVC, the THD voltage at all buses was normalized as its maximum value does not exceed $5 \%$ as shown in figure 10 . The value of the THD voltage exceeds its recommended limits at buses where the nonlinear loads were connected. Moreover, as shown in figure 09, we have noticed that the THD voltage at the SVC bus is still constant in cases 02 and 03 because the SVC location is so far from the location of the nonlinear loads connected at buses 04 and 05, respectively.

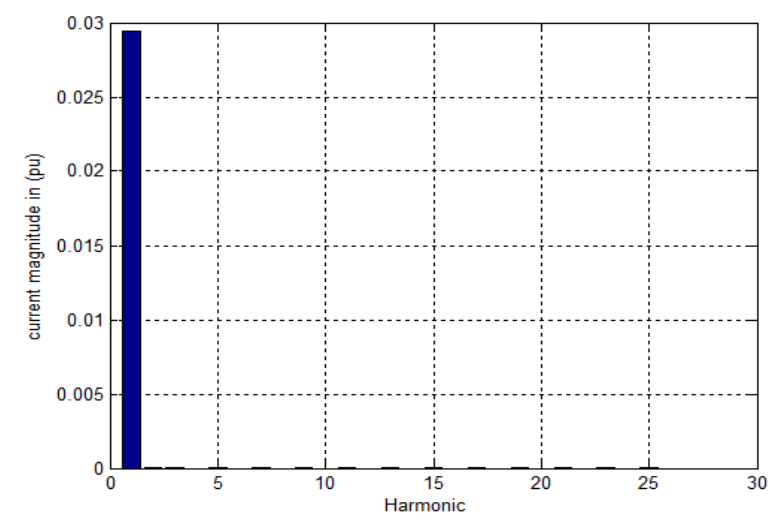

(b)

Figure 6. Harmonic current of the SVC) at alpha $=90^{\circ}($ a)-Waveform, $(b)$ - spectrum 


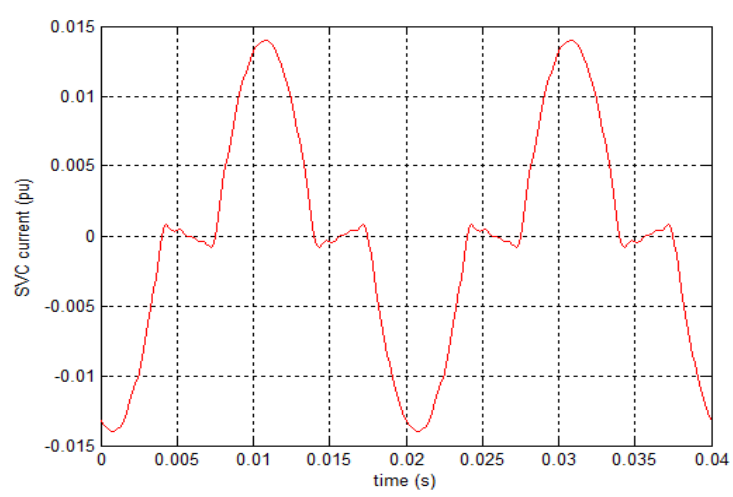

(a)

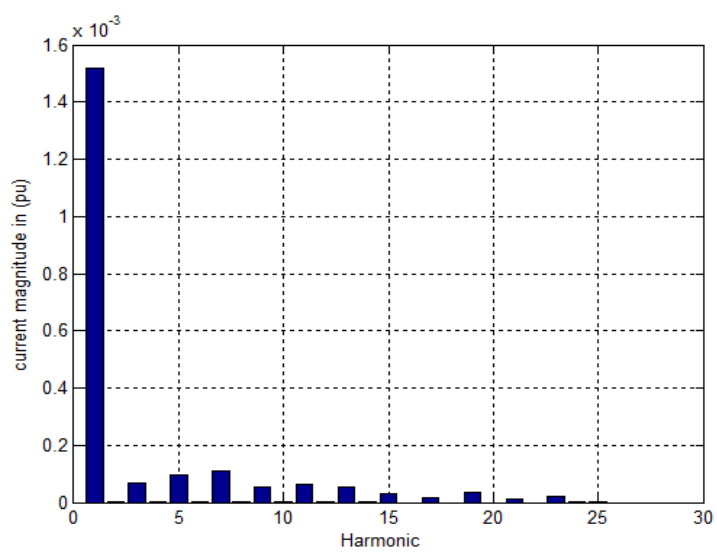

(b)

Figure 7. Harmonic current of the SVC) at alpha $=120^{\circ}($ a)- Waveform, $(b)-$ spectrum

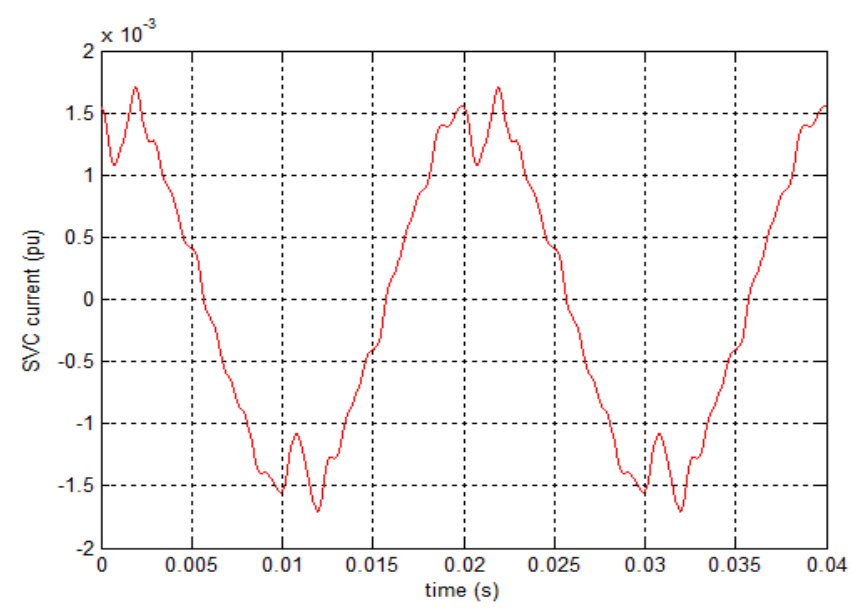

(a)

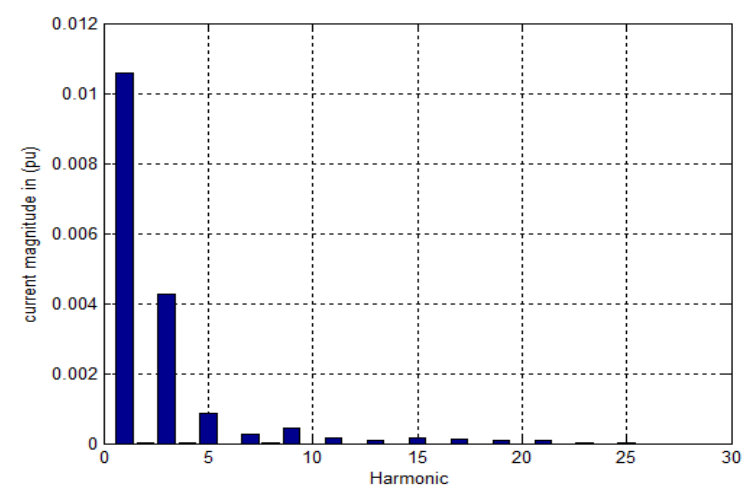

(b)

Figure 8. Harmonic current of the SVC) at alpha $=160^{\circ}($ a)- Waveform, $(b)-$ spectrum

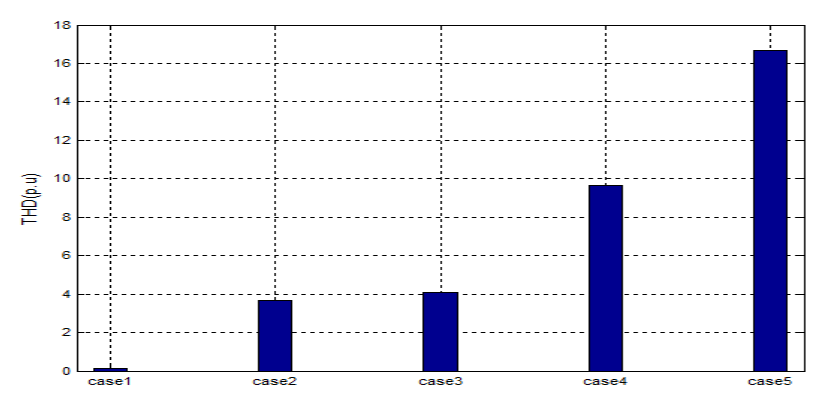

Figure 9. Voltage THD at bus 14

In the case where the nonlinear loads are connected close to the SVC, the THD voltage increases suddenly from about $4 \%$ to $10 \%$ and $16 \%$ for cases 4 and 5 respectively, since this voltage bus carries a high content of harmonics as well as the level of harmonic current injected into system is larger.

Figure 11 gives the harmonic components of the SVC for five cases. The changing in the 8 harmonic components is almost constant for cases 2 and 3. On the other hand, when the nonlinear load is close to the SVC bus, this causes a high distortion in the applied voltage and consequently the harmonic current becomes larger due to the harmonic interaction between the ac system and the SVC. This interaction leads also to an unexpected change in the magnitude for each harmonic order of interest. As indicated in Fig.11. In case 5, the nineteenth component has the highest magnitude. while in cases $01 ; 02 ; 03$ the fifth component had the highest magnitude. 


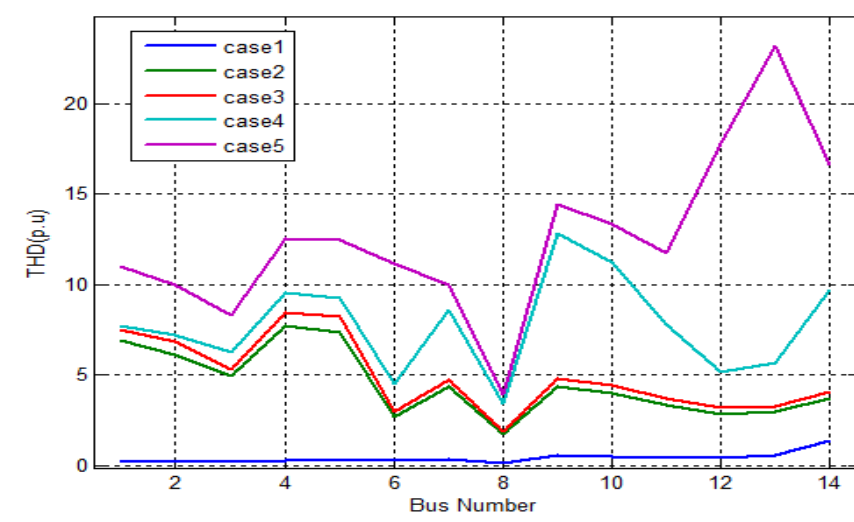

Figure 10. The Voltage THD at all buses for different cases

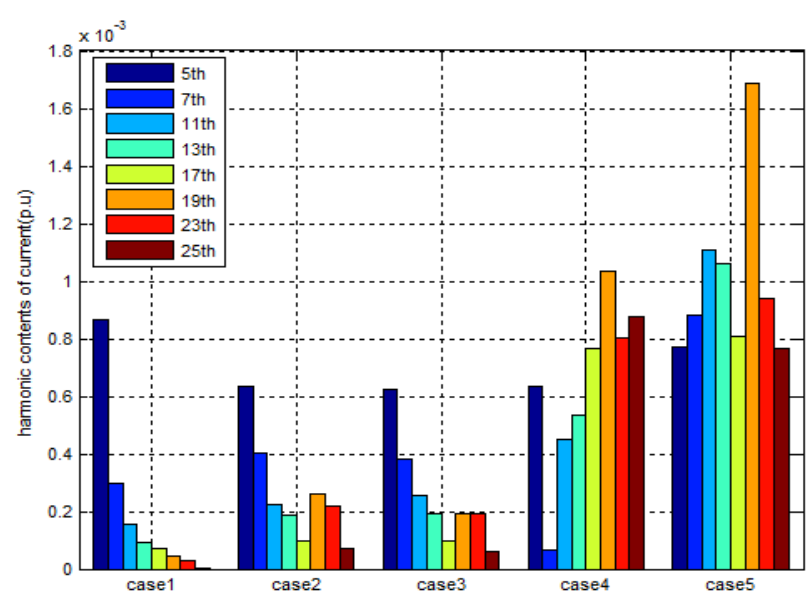

Figure 11. Harmonic current component of the SVC for different cases

\section{Conclusion}

In this paper, a new harmonic analysis formulation of a power system network including the static Var compensator is developed using the Newton Raphson method. The non-linear system equations are developed and the nodal voltages and branch currents are obtained at fundamental and harmonic frequencies. The model of the SVC as coupled harmonic source is developed and included into a harmonic power flow algorithm in order to study the harmonic interaction between the AC system and the SVC devices. According to this study, the SVC voltage waveform changing with delay angle is presented. Moreover, the total harmonic distortion voltage has varied as function of the number and location of the nonlinear load with respect to the SVC bus.
In further work, the best location of the SVC in larger power systems will be performed using intelligent techniques to find out the best SVC sizing and location.

\section{References}

[1] Hingorani, N G., Gyugyi, L., El-Hawary, M. : Understanding FACTS: concepts and technology of flexible AC transmission systems, Wiley Online Library, New York, 2000.

[2] Blasina, M., Komen, V., Renato, Ć. : Voltage control and compensation of reactive power by SVC devices, Engineering review, 30 (2010), 2, 47-58.

[3] Wilkosz, K., Sobierajski, M., Kwasnicki, W. : The analysis of harmonic generation of SVC and STATCOM by EMTDC/PSCAD simulations, 8th International Conference on Harmonics and Quality of Power. Proceedings (Cat. No.98EX227), Athens, Greece, 1998, 853-858.

[4] Fan, Z., Johan, E. : Harmonic Impedance Analysis in the Presence of Static Var Compensator (SVC), 2006 IEEE PES Power Systems Conference and Exposition, Atlanta, GA, USA, 2006, 1485-1492.

[5] Warner, J., Fenn, J., Hutchinson, S., Platt, R. : Impact of system changes on the harmonic performance and rating of an SVC, PES T\&D 2012, Orlando, FL, USA, 2012, 1-7.

[6] Shawon, M H., Hanzelka, Z., Dziadecki, A. : Voltage-current and harmonic characteristic analysis of different FC-TCR based SVC, 2015 IEEE Eindhoven PowerTech, Eindhoven, Netherlands, 2015, 1-6.

[7] Wang, H L., Lin, M S. : A probabilistic approach for SVC placement with harmonic control and reactive power compensation, 2015 IEEE Innovative Smart Grid Technologies Asia (ISGT ASIA), Bangkok, Thailand, 2015, 16.

[8] Bonner, A. et al. : Modeling and simulation of the propagation of harmonics in electric power networks. I. Concepts, models, and simulation techniques, IEEE Transactions on Power Delivery, 11 (1996), 1, 452-465.

[9] Xia, D., Heydt, G T. : Harmonic power flow studies part I-formulation and solution, IEEE Transactions on Power Apparatus and Systems, PAS-101 (1982), 6, 1257-1265.

[10] Xia, D., Heydt , G. : Harmonic power flow studies-part II implementation and practical 
application, IEEE Transactions on Power Apparatus and Systems, PAS-101 (1982), 6, 1266-1270.

[11] Kraimia, M N., Zellagui, M., Boudour, M. : harmonic power flow calculations for algerian electric transmission system with multiple non linear loads, lgerian Large Electrical Network Conference to the Electrical Networks of Future CIGRÉ 2015, Hilton-Algiers-Algeria, 2015, 18.

[12] Kraimia, M N., Zellagui, M., Boudour, M. : Harmonic Analysis on Power System in the Presence of Thyristor Controlled Series Compensation under Non Sinusoidal Condition, 2nd International Conference on Power Electronics and their Applications (ICPEA), Djelfa , Algeria, 2015,

[13] Kraimia, M N., Zellagui, M., Boudour, M. : Impact of Renewable Energy Source Penetration on Total Harmonic Distortion Using Harmonic Power Flow, 1st National Conference on Renewable Energy Technologies and Applications (NCRETA), Guelma, algeria, 2014 ,

[14] Lin, W-M., Zhan, T-S., Tsay, M-T. : Multiplefrequency three-phase load flow for harmonic analysis, IEEE transactions on Power Systems, 19 (2004), 2, 897-904.

[15] Moreno, M Á., Usaola, J. : A new balanced harmonic load flow including nonlinear loads modeled with RBF networks, IEEE Transactions on Power Delivery, 19 (2004), 2, 686-693.

[16] Smith, B., Arrillaga, J. : Power flow constrained harmonic analysis in AC-DC power systems, IEEE transactions on Power Systems, 14 (1999), 4, 1251-1261.

[17] Thunberg, E., Soder, L. : A Norton approach to distribution network modeling for harmonic studies, IEEE Transactions on Power Delivery, 14 (1999), 1, 272-277.

[18] Ulinuha, A. : Application of Decoupled Harmonic Power Flow for Assessment of Harmonic Passive Filter Size and Location, Proceedings of the 2016 International Conference on Industrial Engineering and Operations Management, Kuala Lumpur, Malaysia, 2016, 1507-1514.

[19] Panfilov, D I., Elgebaly, A E. : Modified thyristor controlled reactor for static VAR compensators, 2016 IEEE International Conference on Power and Energy (PECon), Melaka, Malaysia, 2016, 712-717.

[20] Bohmann, L J., Lasseter, R H. : Harmonic interactions in thyristor controlled reactor circuits, IEEE Transactions on Power Delivery, 4 (1989), 3, 1919-1926.

[21] Fuchs, E., Masoum, M A. : Power quality in power systems and electrical machines, Academic Press, 2008.

[22] Freris, L., Sasson, A. : Investigation of the loadflow problem, Proceedings of the institution of electrical engineers, 115 (1968), 10, 1459-1470. 


\section{Appendix}

Table A.1. The harmonic spectrum of nonlinear load connected at bus 12 (scenario 01)

\begin{tabular}{|c|c|c|c|c|c|c|c|c|c|}
\hline $\begin{array}{c}\text { Harmonic } \\
\text { order }\end{array}$ & $\mathbf{1}$ & $\mathbf{5}$ & $\mathbf{7}$ & $\mathbf{1 1}$ & $\mathbf{1 3}$ & $\mathbf{1 7}$ & $\mathbf{1 9}$ & $\mathbf{2 3}$ & $\mathbf{2 5}$ \\
\hline MAg (\%) & 100 & 18.24 & 11.9 & 5.73 & 4.01 & 1.93 & 1.39 & 0.94 & 0.86 \\
\hline $\mathbf{P h}\left({ }^{\circ}\right)$ & 0 & -55.68 & -84.11 & -143.56 & -175.5 & 111.39 & 68.3 & -24.61 & -67.64 \\
\hline
\end{tabular}

Table A.2. The harmonic spectrum of nonlinear loads (scenario 02)

\begin{tabular}{|c|c|c|c|c|c|c|c|c|c|c|}
\hline \multirow{2}{*}{$\begin{array}{c}\text { Harmonic } \\
\text { order }\end{array}$} & \multicolumn{2}{|c|}{ NLD at Bus 04 } & \multicolumn{2}{l|}{ NLD at Bus 05 } & \multicolumn{2}{l|}{ NLD at Bus 09 } & \multicolumn{2}{l|}{ NLD at Bus 11 } & \multicolumn{2}{l|}{ NLD at Bus 13 } \\
\cline { 2 - 12 } & $\begin{array}{c}\text { Mag } \\
(\%)\end{array}$ & $\begin{array}{c}\text { Ph } \\
\left({ }^{\circ}\right)\end{array}$ & $\begin{array}{c}\text { Mag } \\
(\%)\end{array}$ & $\begin{array}{c}\text { Ph } \\
\left({ }^{\circ}\right)\end{array}$ & $\begin{array}{c}\text { Mag } \\
(\%)\end{array}$ & $\begin{array}{c}\text { Ph } \\
\left({ }^{\circ}\right)\end{array}$ & $\begin{array}{c}\text { Mag } \\
(\%)\end{array}$ & $\begin{array}{c}\text { Ph } \\
\left({ }^{\circ}\right)\end{array}$ & $\begin{array}{c}\text { Mag } \\
(\%)\end{array}$ & $\begin{array}{c}\text { Ph } \\
\left({ }^{\circ}\right)\end{array}$ \\
\hline $\mathbf{1}$ & 100 & 0 & 100 & 0 & 100 & 0 & 100 & 0 & 100 & 0 \\
\hline $\mathbf{5}$ & 18.24 & -55.68 & 4.24 & -95.68 & 20 & 0 & 23.52 & 111 & 82.8 & -135 \\
\hline $\mathbf{7}$ & 11.9 & -84.11 & 17.9 & -74.11 & 14.3 & 0 & 6.08 & 109 & 77.5 & 69 \\
\hline $\mathbf{1 1}$ & 5.73 & -143.56 & 8.3 & -113.56 & 9.1 & 0 & 4.57 & -158 & 46.3 & -62 \\
\hline $\mathbf{1 3}$ & 4.01 & -175.5 & 7.01 & -194.58 & 7.7 & 0 & 4.2 & -178 & 41.2 & 139 \\
\hline $\mathbf{1 7}$ & 1.93 & 111.39 & 1.93 & 11.39 & 5.9 & 0 & 1.8 & -94 & 14.2 & 9 \\
\hline $\mathbf{1 9}$ & 1.39 & 68.3 & 2.39 & 38.3 & 5.3 & 0 & 1.37 & -92 & 9.7 & -155 \\
\hline $\mathbf{2 3}$ & 0.94 & -24.61 & 0.64 & -4.61 & 4.3 & 0 & 0.75 & -70 & 1.5 & -158 \\
\hline $\mathbf{2 5}$ & 0.86 & -67.64 & 0.46 & -17.64 & 4 & 0 & 0.56 & -70 & 2.5 & 98 \\
\hline
\end{tabular}

